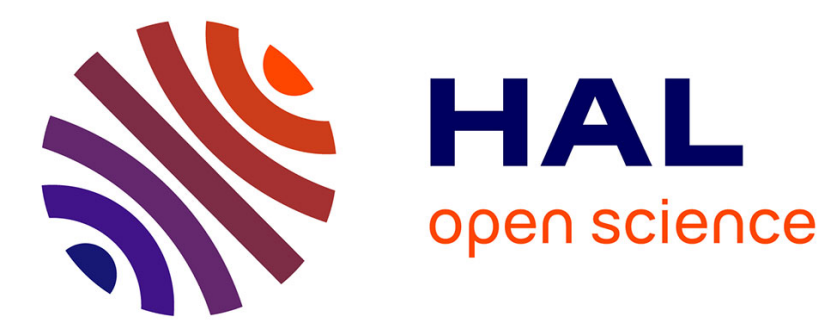

\title{
Red Alert: a cognitive countermeasure to mitigate attentional tunneling
}

Julie Saint-Lot, Jean-Paul Imbert, Frédéric Dehais

\section{To cite this version:}

Julie Saint-Lot, Jean-Paul Imbert, Frédéric Dehais. Red Alert: a cognitive countermeasure to mitigate attentional tunneling. CHI '20 - 38th SIGCHI conference on Human Factors in computing systems, Apr 2020, Honolulu, Hawaï, United States. pp.1-6, 10.1145/3313831.3376709 . hal-02882108

\section{HAL Id: hal-02882108 \\ https://hal.science/hal-02882108}

Submitted on 26 Jun 2020

HAL is a multi-disciplinary open access archive for the deposit and dissemination of scientific research documents, whether they are published or not. The documents may come from teaching and research institutions in France or abroad, or from public or private research centers.
L'archive ouverte pluridisciplinaire HAL, est destinée au dépôt et à la diffusion de documents scientifiques de niveau recherche, publiés ou non, émanant des établissements d'enseignement et de recherche français ou étrangers, des laboratoires publics ou privés. 


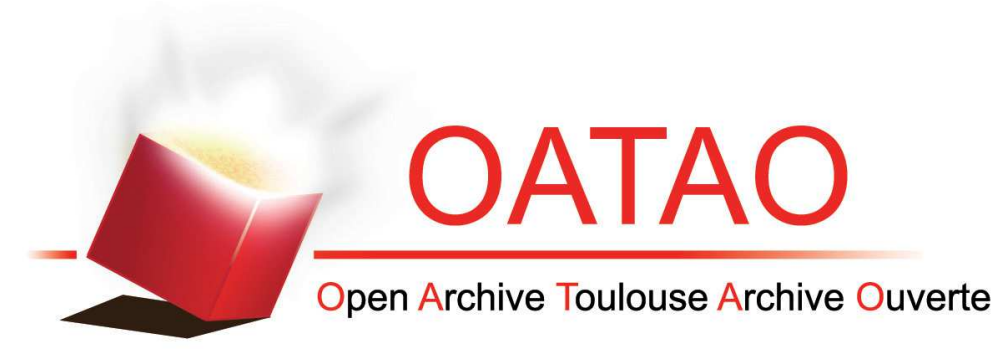

\section{Open Archive Toulouse Archive Ouverte (OATAO)}

OATAO is an open access repository that collects the work of some Toulouse researchers and makes it freely available over the web where possible.

This is an author's version published in: https://oatao.univ-toulouse.fr/25742

Official URL:https://doi.org/10.1145/3313831.3376709

\section{To cite this version :}

Saint-Lot, Julie and Imbert, Jean-Paul and Dehais, Frédéric Red Alert: a cognitive countermeasure to mitigate attentional tunneling. (2020) In: The ACM Conference on Human Factors in Computing Systems - CHI 2020, 25 April 2020 - 30 April 2020 (Honolulu, Hawaï, United States).

Any correspondence concerning this service should be sent to the repository administrator: tech-oatao@listes-diff.inp-toulouse.fr 


\section{Red Alert: a cognitive countermeasure to mitigate attentional tunneling}

\author{
Julie Saint-Lot \\ ENAC, Université de Toulouse, \\ France \\ julie.saint-lot@enac.fr
}

\author{
Jean-Paul Imbert \\ ENAC, Université de Toulouse, \\ France \\ jean-paul.imbert@enac.fr
}

\author{
Frédéric Dehais \\ ISAE Supaéro, Université de \\ Toulouse, France \\ frederic.dehais@isae-supaero.fr
}

\begin{abstract}
Attentional tunneling, that is the inability to detect unexpected changes in the environment, has been shown to have critical consequences in air traffic control. The motivation of this study was to assess the design of a cognitive countermeasure dedicated to mitigate such failure of attention. The Red Alert cognitive countermeasure relies on a brief orange-red flash $(300 \mathrm{~ms})$ that masks the entire screen with a $15 \%$ opacity. Twenty-two air traffic controllers faced two demanding scenarios, with or without the cognitive countermeasure. The volunteers were not told about the Red Alert so as to assess the intuitiveness of the design without prior knowledge. Behavioral results indicated that the cognitive countermeasure reduced reaction time and improved the detection of the notification when compared to the classical operational design. Further analyses showed this effect was even stronger for half of our participants $(91.7 \%$ detection rate) who intuitively understood the purpose of this design.
\end{abstract}

\section{Author Keywords}

Air traffic control; notification; interruption; attentional tunneling; countermeasure; Air Traffic Controller (ATCO).

\section{CSS Concepts}

Human-centered computing $\sim$ Human computer interaction (HCI), HCI design and evaluation methods; User studies; Laboratory experiments

\section{INTRODUCTION}

Air traffic control is a challenging task that takes place under a dynamic and uncertain environment. Air Traffic Controllers (ATCO) have to supervise air traffic on their radar while communicating with pilots to ensure the safety and fluidity of traffic. This activity requires focused and divided attention abilities to process local information while remaining responsive to peripheral and unexpected events such as conflicts (i.e. potential collisions) [6]. It is now well admitted that high workload (e.g. density and complexity of air traffic) may negatively impact attentional performance (C) 2020 Association for Computing Machinery. ACM acknowledges that this contribution was authored or co-authored by an employee, contractor or affiliate of a national government. As such, the Government retains a nonexclusive, royalty-free right to publish or reproduce this article, or to allow others to do so, for Government purposes only.

CHI 2020, April 25-30, 2020, Honolulu, HI, USA

(C) 2020 Association for Computing Machinery.

ACM ISBN $978-1-4503-6708-0 / 20 / 04 \ldots \$ 15.00$

https://doi.org/10.1145/3313831.3376709 to an extent that ATCO can fail to perceive critical events [18]. Different "warning" and "alert" notifications have been developed to overcome such attentional impairment while keeping the human operator in the loop. Warnings that have lower levels of urgency are displayed on the radar labels with static red text, while more critical alerts currently use blinking colored text. For example, a ShortTerm Conflict Alert (STCA) is triggered when an aircraft does not meet the separation criteria (5 nautical mile longitudinal, $1000 \mathrm{ft}$ altitude), leading to a flashing red 'ALRT' notification on the aircraft track label (cf. figure 3up). Despite these technical improvements, a recent study disclosed that STCA could remain undetected [12], thus confirming the findings of the French Board for Safety Event analysis (ITES). Indeed, in 2013, the ITES Human Factors Group identified failure of attention as a possible issue and recommended to enhance the design of the critical alarms because of several events linked to the absence of response to alert notifications.

A relevant approach to improve the design of alert notifications is to understand the mechanisms underpinning failure of attention. Different models have been put forth to account for this phenomenon such as a shrinkage of the functional field of view, also known as "visual tunneling" [22], or dilution of visual attention, also known as "general interference" [8]. Consistently with these hypotheses, some studies have shown that, depending on the experimental task, the detection of peripheral stimuli was adversely affected if they were placed at 2.2 degrees [22], 7 degrees [8] or 10 degrees of eccentricity from foveal field [15]. Vision research may provide interesting recommendations such as the use of motion [4] and specific colors (e.g. yellow) [1] to increase the likelihood of attentional capture. Luminance, size and frequency of the signal also represent potential relevant features to be considered [3]. However, improving the saliency of the alert does not guarantee that it will attract attention and reach awareness. For instance, several experimental studies have revealed that salient but unexpected events can remain undetected even when presented in the foveal field $[5,14,19]$. This inattentional blindness phenomenon also known as attention tunneling [21] results from subtle interactions between two correlated neural networks, namely the dorsal and ventral pathways. The ventral pathway is dedicated to process new stimuli in a bottom-up fashion, whereas the dorsal pathways imposes a top-down control to focus attention toward task at hand 
related stimuli. However, under demanding settings, this latter pathway may take over the ventral one, thus preventing disengagement, shifting and re-orientation of attention to process new incoming stimuli [20].

One efficient way to mitigate attentional tunneling is to design cognitive countermeasures. Rather than adding alarms, one solution consists in temporarily removing the information (e.g. display) on which the human operator is excessively focusing and to replace it by an explicit notification in their visual field [9]. Therefore, the user interface acts as a cognitive prosthesis as it performs the attentional disengagement, shifting and re-orientation of attention. These cognitive countermeasures were successfully tested with pilots [10] and with unmanned vehicle operators during attentional tunneling episodes. However, this design may not be appropriate for all contexts and it should be considered only in extreme cases when there is a need to instigate a change in human operator strategy [11]. Indeed, such countermeasure should not be considered as another form of alert. For instance, [12] demonstrated that this information removal drastically reduced interruption lag (i.e. reaction time to interrupt the primary task to process the alarm) but led to higher "resumption lag" (recovery time to resume primary task). These considerations demonstrate the complexity of designing appropriate solution to mitigate failure of attention.

The objective of the present study is to implement a new design inspired by the work of [9, 10] and [12]. This countermeasure masks the entire screen for $300 \mathrm{~ms}$ using red-orange flash with an opacity of $15 \%$, in addition to the operational design of notifications. In contrast to [9, 10], this countermeasure does not consist of an actual information removal, because the aircraft call signs and radar frames remain perfectly visible. The design was tested with 22 experienced ATCO in the LABY micro-world [13] facing highly demanding scenarios with or without the countermeasure. Participants were not told about the use of the countermeasure so as to assess its immediate effect on attentional abilities and acceptability without prior training.

\section{MATERIAL AND METHOD}

\section{Participants}

Twenty-two experienced air traffic controllers were recruited (10 women and 12 men, mean age: 43 years, mean years of expertise: 15 years).

\section{Experimental platform and scenarios}

The LABY microworld [13] was used to conduct the experiment on a 30 inch-screen (resolution $2560 * 1600$ pixels) similar to the operational ones. This immersive environment simulates key features of ATCO activity with a high degree of realism in terms of visualization and interactions while providing metrics about their behavior. Participants had to guide one or several aircraft in dedicated paths (grey corridor on figure 1, flows to integrate), avoiding potential conflicts with other aircraft in the vicinity by setting adequate speed/level/heading (c.f. figure 2 ). Aircraft were to be separated in distance and altitude and be kept along their path until reaching a specific area (see red square, representing the runway on figure 1). Participants had to click on any aircraft with the critical STCA alert (see figure 1, right bottom corner) to notify their detection and then to manage the conflictual trajectory. The LABY scenario is built with an editor which allows to design the routes and the events to be triggered. Metrics such as reaction time and accuracy on events and compliance of aircraft on the routes are collected.

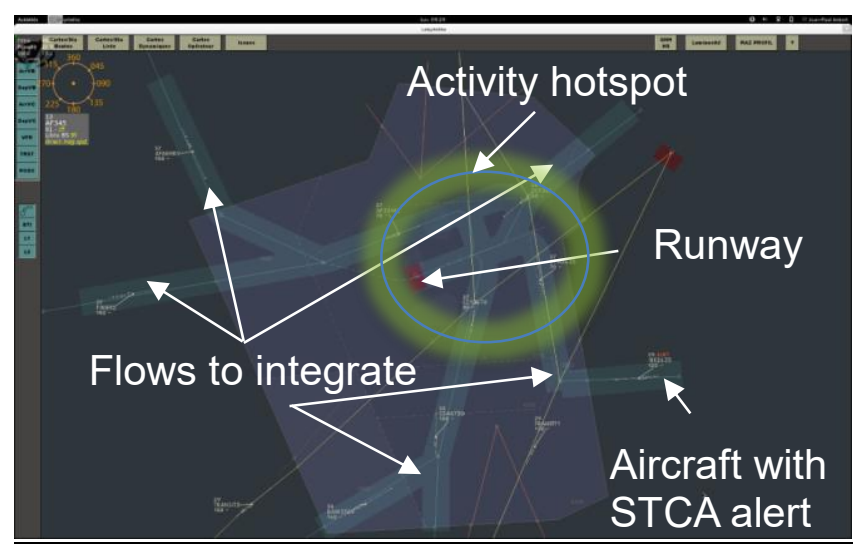

Figure 1: LABY screen configured for our experiment

In this experiment, the LABY micro-world was configured to replicate the task of an approach ATCO (Arrivals) on a virtual airport commonly used for training. This activity is prone to attentional tunneling since the ATCO has to integrate multiple flows on the runway axis while maintaining separation minima and give altitude orders to intercept the landing system. The green circle labelled activity hotspot on figure 1 shows where most of ATCO's activity was located according to the airport activity.

Two challenging scenarios were designed and pre-tested with expert ATCO. They involved medium to heavy traffic situations dedicated to induce episodes of high mental demands and attentional tunneling. Participants were required to supervise and guide incoming aircraft through six grey corridors surrounding the runway.

Six STCA alerts were automatically triggered during each scenario on aircraft and participants were required to acknowledge them as fast as possible and handle the conflictual trajectory. In one of the scenarios, the participants were assisted with the Red Alert cognitive countermeasure whereas in the other one not. 


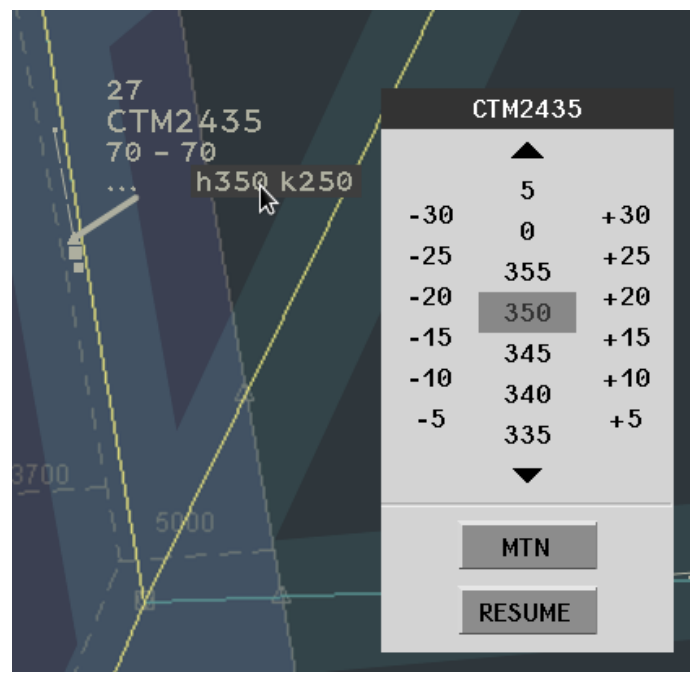

Figure 2: LABY label and heading menu (selected value $350^{\circ}$ )

\section{Red Alert cognitive countermeasure design}

The design of the cognitive countermeasure consisted of a $300 \mathrm{~ms}$ orange-red flash that covering the entire screen with a $15 \%$ opacity. All tactical information displayed on the screen remained visible during the flash.
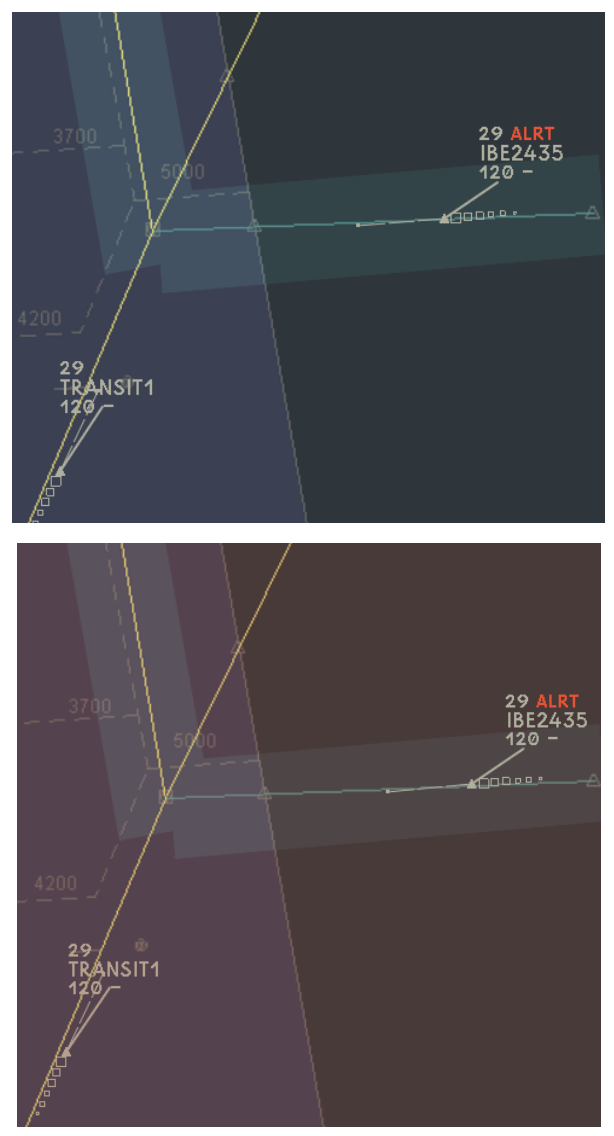

Figure 3: Up - Current notification alert design: a blinking red "ALRT" message is displayed on the aircraft label. Down Red Alert countermeasure design: the entire screen is masked with a $15 \%$ opacity red-orange layer for $300 \mathrm{~ms}$.

\section{Procedure}

Participants sat in a comfortable chair and began to read the instructions to interact with LABY (c.f. figure 4). They then performed a training exercise to familiarize themselves with the use of the micro-world. Once the training completed, the volunteers performed the 2 scenarios (15 minutes each). Six STCA alerts occurred during each scenario. One scenario was presented with the Red Alert countermeasure (CM) while the other wasn't (No CM). The presence or absence of the cognitive countermeasure in one of the two scenarios was done in random fashion to control for potential training and fatigue effects.

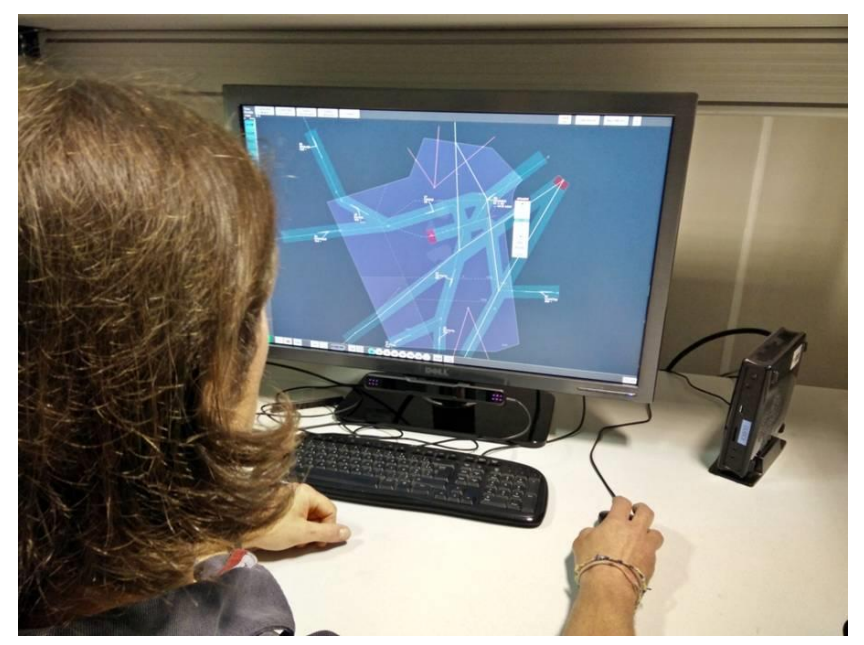

Figure 4: Experimental setup

\section{Measurements}

\section{Subjective measures}

After each scenario, the participants had to fill in the NASA TLX to assess their level of mental workload. At the end of the experiment, a direct interview was conducted and the following questions were asked: did you perceive the orange-red flashes, did you understand their purpose and were they efficient to alert you.

\section{Objective measures}

In order to assess the efficiency of the red alert cognitive countermeasure, the mean rate of detected STCA alerts, the mean time to acknowledge theses alert and the mean performance on the primary ATCO task (i.e. guiding and maintaining the aircraft within the corridor limits) were measured. All statistical analyses were carried out using the $R$ software. The p-value threshold for significance was set to 0.05 if not otherwise mentioned. When appropriate, posthoc comparisons were carried out using the Tukey's Honestly Significant Difference test.

\section{RESULTS}

\section{Subjective results}

A paired t-test (two tailed) disclosed no significant effect of the scenario type (CM vs No CM) on workload as measured by NASA-TLX: $t(21)=0.10, p=0.9$. 
Post experiment debriefing revealed that half of the participants $(N=11)$ understood the purpose of the cognitive countermeasure. These participants declared that the Red Alert enhanced their ability to perceive the STCA notification. The other half $(N=11)$ reported that they did not understand the purpose of the cognitive countermeasure: they declared that they perceived it but thought that it was either a failure of the LABY software or the screen or that the red flash was dedicated to distracting their attention intentionally to increase stress.

\section{Behavioral results}

We first examined the effect of the design type (Red Alert cognitive countermeasure vs classical operation design) on STCA alarm perception. A paired t-test (two tailed) (CM, no $\mathrm{CM}$ ) disclosed that the participants significantly detected more alarms with the red alert $(M=69.4 \%, S D=28.6 \%)$ than with the classical operational design ( $M=57.9 \%$, $S D=26.4 \%): t(21)=2.13, p<0.05-$ see figure 5 .

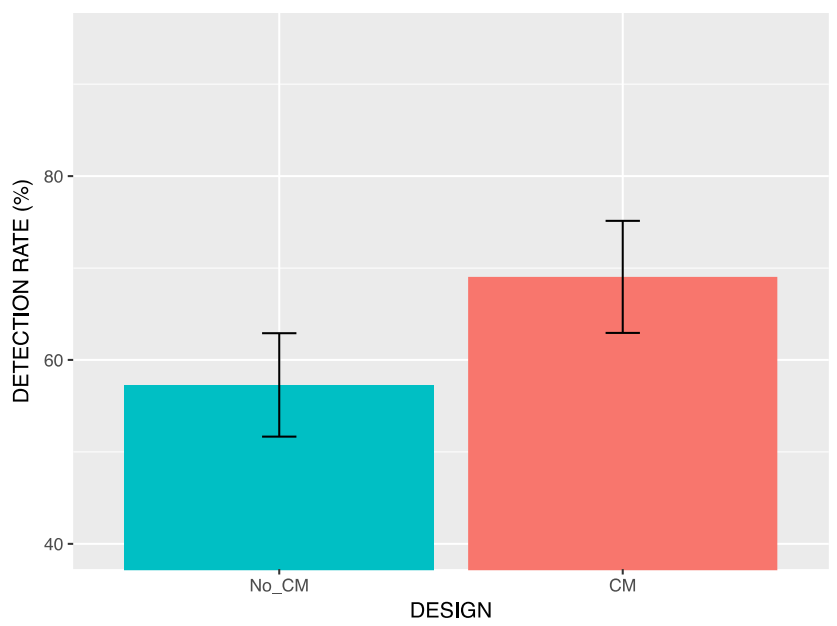

Figure 5: STCA alarm mean detection rate without the cognitive countermeasure (left, "No_CM") and with the cognitive countermeasure (right, "CM"). Bars represent standard error

Further analyses were conducted to investigate potential behavioral differences between the group of participants who understood the Red Alert cognitive countermeasure and the one who did not. The two-way ANOVA 2 (CM vs NO CM; Group who understood vs Group who did not understood) disclosed a significant CM $\mathrm{x}$ GROUP interaction effect: $F(1,20)=14.01, p=0.001, \eta^{2} p=0.12$. Posthoc analysis revealed that participants who understood the cognitive countermeasure perceived more alarms in the CM scenario $(M=91.7 \%, S D=10.6 \%)$ than the group who did not understand the cognitive countermeasure $(M=47.0 \%$, $S D=23.6 \%$ ) - see figure 6 .

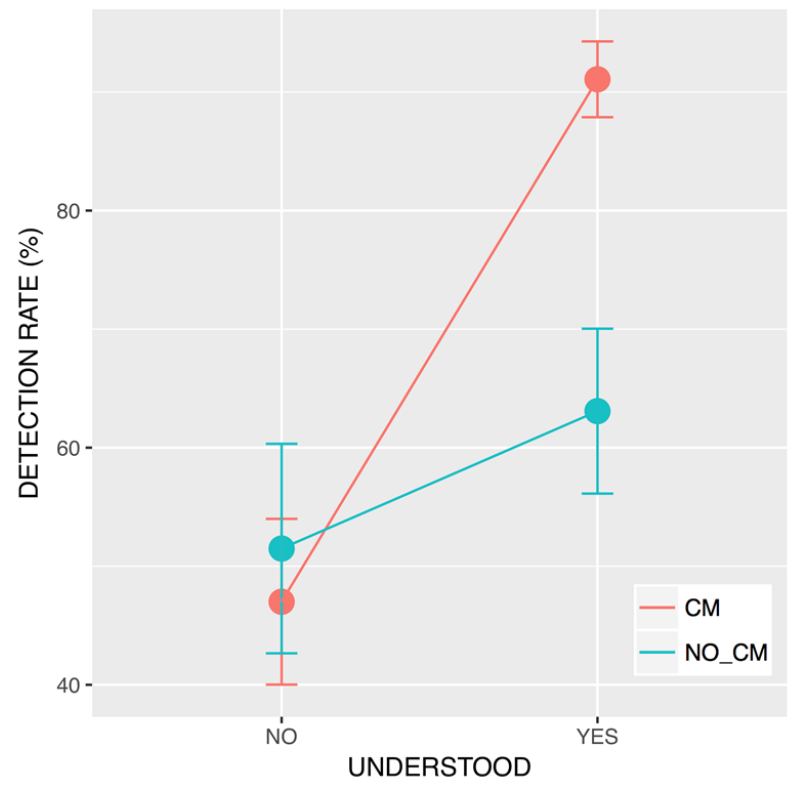

Figure 6: Impact of the presence of the cognitive countermeasure on alarm detection rate depending on the group type (i.e. group who understood vs group who did not understand the cognitive countermeasure). This figure shows that the cognitive countermeasure improved the performance of the group who intuitively understood the cognitive countermeasure. Bars represent standard error.

Then analysis to investigate the effect of the design type (red alert cognitive countermeasure vs classical operation design) was conducted on reaction time to detect the STCA alert. The statistical analyses revealed that the participants had significant lower mean response time in the CM scenario $(M=6.4 \mathrm{~s}, S D=4.1 \mathrm{~s})$ than in the No-CM $(M=9.7 \mathrm{~s}$, $S D=5.7 \mathrm{~s}): t(21)=-2.50, p<0.05 s-$ see figure 7.

We then ran further analyses to investigate potential differences between the mean reaction time of the group of participants who understood the red alert cognitive countermeasure and the one who did not. The two-way ANOVA 2 (CM vs NO CM; Group who understood vs Group who did not understand) disclosed a main effect of the group (understanding vs non understanding): $F(1,20)=$ 17.92, $p<0.001, \eta^{2} p=0.29$ and of the design type (CM vs no CM): $F(1,20)=5.96387825, p<0.01, \eta^{2} p=0.14$. However this ANOVA did not reveal a significant CM x GROUP interaction effect: $F(1,20)=0.7, p=0.8, \eta^{2} p=0.01$ and thus did not allow us to run post-hoc analyses but only to perform descriptive statistics. The participants who understood the cognitive countermeasure exhibited faster mean reaction time in the $\mathrm{CM}$ scenario $(M=3.6 \mathrm{~s}, S D=$ $2.62 \mathrm{~s}$ ) than the group who did not understand the cognitive countermeasure $(M=9.5 s, S D=5.9 s)$. 


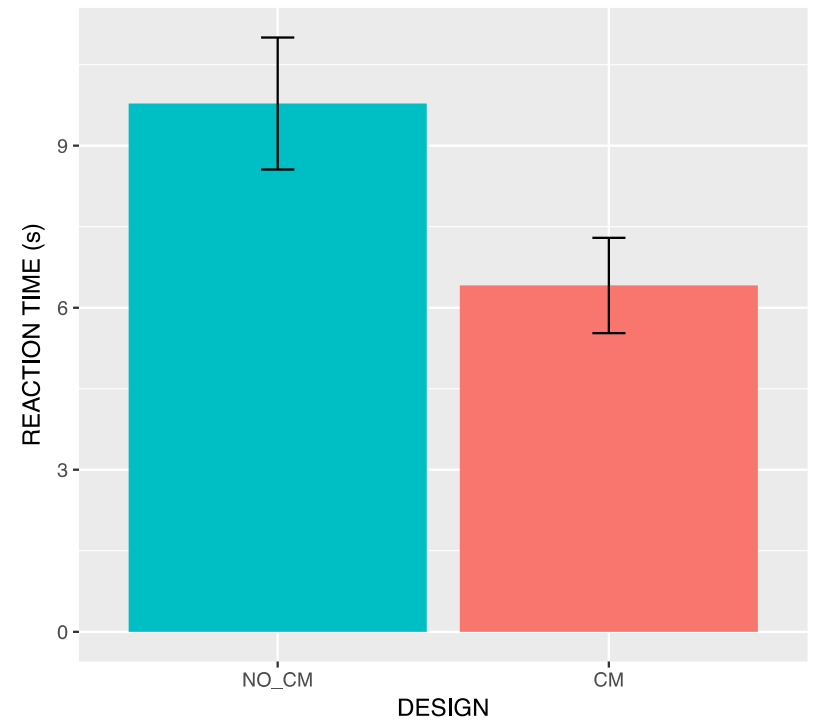

Figure 7: Mean reaction time (in s) to detect the STCA alert without the cognitive countermeasure (left, "No_CM") and with the cognitive countermeasure (right, "CM"). Bars represent standard error.

Finally, we conducted analysis to investigate the effect of the design type on the primary ATC task (i.e. guiding the aircraft within the corridor). The statistical analysis revealed no significant effect: $F(1,20)=2.1, \quad p=0.16$, $\eta^{2} p=0.09$. Further analysis did not disclose any significant difference between the group who understood the red alert cognitive countermeasure and the group who did not.

\section{DISCUSSION}

The objective of this study was to implement a cognitive countermeasure, dedicated to mitigate attentional tunneling in the context of air traffic control. Based on previous studies $[9,10,12]$, a new design based on the display of an orange-red flash with a $15 \%$ opacity masking the entire screen for $300 \mathrm{~ms}$ was proposed. The rationale was to induce attentional disengagement from the primary task and to shift and re-orient the locus of attention onto the STCA alert. The color of the cognitive countermeasure is the same as the STCA alert notification so as to induce a priming effect [7]. The efficiency of this design was tested by placing ATCO in a realistic situation under demanding attentional setting.

The results of this experiment revealed that our scenarios were efficient to induce attentional tunneling as our participants missed almost $42 \%$ of STCA alerts in the scenario without countermeasure. When perceived, STCA alerts led to relatively long reaction time $(\sim 10 \mathrm{~s})$. This study confirms previous findings [12] and conclusions from the French Board for Safety events analysis that failure of attention can take place in the context of ATC even with highly trained controllers. Consistently with the hypotheses, the analyses disclosed that the use of the cognitive countermeasure significantly improved detection rate of the alert while reducing reaction time when understood.

Interestingly enough, the Red Alert cognitive countermeasure did not decrease the performance on the primary task (i.e. guiding the aircraft within the corridor). This is an important point as [12] showed that a previous design based on information removal, while inducing higher alarm detection rate, had a deleterious effect on the primary task performance when compared to the classical operational design. Moreover, one could expect that the cognitive countermeasure led to lower mental workload as measured by NASA-TLX. The results did not disclose such evidences. A plausible explanation is that the subjective rating of the participants only account for the management of the challenging primary task no matter the presence or absence of the cognitive countermeasure. The brief occurrence of only six STCA/cognitive countermeasures was not enough to modulate the global perceived workload.

One other research question was to assess whether this new design could be intuitively processed without any training. The post-experiment debriefing session revealed that half of our participants intuitively understood the cognitive countermeasure whereas the others declared it confused them. When looking at the performance of these two groups, it appeared that the one who positively reacted to the cognitive countermeasure almost missed no alerts and showed a reaction time reduced by a factor of 2.6 when compared to the scenario without the countermeasure. The group who did not understand the cognitive countermeasure did not exhibit behavioral differences with or without the Red Alert countermeasure. These findings indicate that training is necessary since the design does not direct the gaze towards a specific area. However, the high performance of the group who understood the cognitive countermeasure shows that it can lead to great efficiency and considerably improve operational safety.

Future work should focus on conducting more experiments with a higher sample of participants facing different types of scenarios to confirm our findings. Eventually, one should consider potential habituation effects if the occurrence of the Red Alert becomes high. The use of a GLSL shader could be considered to overcome this issue as the alert images will be designed in a slightly different way each time it is presented to the ATCO. Eventually, the integration of eye tracking $[16,17]$ and portable monitoring device [11] open promising perspective to detect failure of attention in real time so as to close the loop and trigger cognitive countermeasure in an adaptive fashion [2].

\section{ACKNOWLDEGMENTS}

We thank all the volunteers who took part in the experiment. 


\section{REFERENCES}

[1] Ancman, E. I. L. E. E. N. 1991. Peripherally located CRTs: Color perception limitations. In Proceedings of the IEEE 1991 National Aerospace and Electronics Conference NAECON 1991 (pp. 960-965).

[2] Aricò, P., Borghini, G., Di Flumeri, G., Colosimo, A., Bonelli, S., Golfetti, Pozzi, S., Imbert, J.P., Granger, G., Benhacene, R., \& Babiloni, F.. 2016. Adaptive automation triggered by EEG-based mental workload index: a passive brain-computer interface application in realistic air traffic control environment. Frontiers in human neuroscience, 10, 539.

[3] Athènes, S., Chatty, S., \& Bustico, A. 2000. Human factors in ATC alarms and notifications design: an experimental evaluation. In Proceedings of the USA/Europe Air Traffic Management R\&D Seminar (pp. 1119-1134).

[4] Bartram, L., Ware, C., \& Calvert, T. 2001. Moving Icons: Detection and Distraction. In INTERACT (Vol. 1, pp. 157-165).

[5] Cartwright-Finch, U., \& Lavie, N. 2007. The role of perceptual load in inattentional blindness. Cognition, 102, 321-340.

[6] Claverie, B., Dubroca, M. C., Ripon, A., \& Paty, J.1986. Etude psychophysiologique des processus attentionnels complexes des officiers contrôleurs de la Circulation Aérienne. Le Travail Humain, 249-260.

[7] Corbetta, M., Miezin, F.M., Dobmeyer, S., Shulman, G.L., \& Petersen. 1990. Attentional Modulation of Neural Processing of Shape, Color, and Velocity in Humans. Science 248.4962, 1556.

[8] Crundall, D., Underwood, G., \& Chapman, P. .1999. Driving experience and the functional field of view. Perception, 28(9), 1075-1087.

[9] Dehais, F., Tessier, C., \& Chaudron, L. 2003. GHOST: experimenting conflicts countermeasures in the pilot's activity. International Joint Conference on Artificial Intelligence, 18, 163-168.

[10] Dehais, F., Causse, M., \& Tremblay, S. .2011. Mitigation of Conflicts with Automation use of cognitive countermeasures. Human Factors: The Journal of the Human Factors and Ergonomics Society, 53(5), 448-460.

[11] Dehais, F., Hodgetts, H. M., Causse, M., Behrend, J., Durantin, G., \& Tremblay, S.. 2019. Momentary lapse of control: A cognitive continuum approach to understanding and mitigating perseveration in human error. Neuroscience \& Biobehavioral Reviews.

[12] Imbert, J.P., Hodgetts, H.M., Parise, R., Vachon, F., Dehais F., \& Tremblay, S. 2014. Attentional costs and failures in air traffic control notifications. Ergonomics Vol. 57, No. 12, 1817-1832. doi: 10.1080/00140139.2014.952680
[13] Imbert, J.P., Hodgetts, H.M., Parise, R., Vachon, F., \& Tremblay, S.. 2014. The LABY microworld: a platform for research, system engineering, and training in air traffic control. In Proceedings of the Human Factors and Ergonomics Society Annual Meeting vol. 58 no. 1 (pp. 1038-1042).

[14] Pappas, J.M., Fishel, S.R., Moss, J.D., Hicks, J.M., \& Leech, T.D.. 2005. An eye-tracking approach to inattentional blindness. In Proceedings of the Human Factors and Ergonomics Society Annual Meeting vol. 49 no. 17 (pp. 1658-1662). doi: $10.1177 / 154193120504901734$

[15] Plainis, S., Murray, I. J., \& Chauhan, K.. 2001. Raised visual detection thresholds depend on the level of complexity of cognitive foveal loading. Perception, 30(10), 1203-1212.

[16] Regis, N., Dehais, F., Tessier, C., \& Gagnon, J.F.. 2012. Ocular metrics for detecting attentional tunneling. In Human Factors and Ergonomics Society Europe Chapter Annual Meeting. Toulouse, France.

[17] Regis, N., Dehais, F., Rachelson, E., Thooris, C., Pizziol, S., Causse, M., \& Tessier, C.. 2014. Formal Detection of Attentional Tunneling in Human Operator-Automation Interactions. IEEE Transactions on Human-Machine Systems (pp. 1-11).

[18] Richard, J.F.. 1980. L'attention. Paris : PUF.

[19] Simons, D. J., \& Chabris, C. F.. 1999. Gorillas in our midst: sustained inattentional blindness for dynamic events. Perception 28, 1059-1074.

[20] Vossel, S., Geng, J. J., \& Fink, G. R.. 2014. Dorsal and ventral attention systems: distinct neural circuits but collaborative roles. The Neuroscientist, 20(2), 150-159.

[21] Wickens, C.D., \& Alexander, A.L.2009. Attentional tunneling and task management in synthetic vision displays. The international journal of aviation psychology, 19(2), 182-199. doi: 10.1080/10508410902766549

[22] Williams, L..- 1982. Cognitive load and the functional field of view. Human Factors: The Journal of the Human Factors and Ergonomics Society, 24(6), 683692. 